\title{
DERIVATIVES OF COMPOSITE FUNCTIONS
}

JOHN RIORDAN

1. Introduction. The object of this note is to show the relation of the $Y$ polynomials of E. T. Bell [1], ${ }^{1}$ first to the formula of diBruno for the $n$th derivative of a function of a function, then to the more general case of a function of many functions. The subject belongs to the algebra of analysis in the sense of Menger [4]; all that is asked is the relation of the derivative of the composite function to the derivatives of its component functions when they exist and no questions of analysis are examined.

2. Function of a single function. Following Dresden [3], take the composite function in the form:

$$
F(x)=f[g(x)]
$$

and for convenience write:

$$
D_{x}^{8} F(x) \equiv F_{s}, \quad\left[D_{u}^{s} f(u)\right]_{u=g(x)} \equiv f_{s}, \quad D_{x g}^{8} g(x) \equiv g_{s},
$$

with $D_{x}=d / d x$.

Then, the first few derivatives of $F(x)$ are as follows:

$$
F_{1}=f_{1} g_{1}, \quad F_{2}=f_{1} g_{2}+f_{2} g_{1}^{2}, \quad F_{3}=f_{1} g_{3}+3 f_{2} g_{2} g_{1}+f_{3} g_{1} .
$$

If these are generalized to

$$
F_{n}=\sum_{i=1}^{n} F_{n, i} f_{i}
$$

the coefficients $F_{n, i}$ are dependent only on the derivatives $g_{1}$ to $g_{i}$, and hence may be determined by specialization of $f$. A convenient choice used by Schlömilch [6] is $f(g)=\exp (a g)$, so that

$$
f_{i}=a^{i} \exp a g
$$

and

$$
e^{-a g} F_{n}=e^{-a g} D_{x}^{n a g} e^{a g}=\sum_{i=1}^{n} F_{n, i}\left(g_{1}, \cdots, g_{i}\right) a^{i},
$$

a generating identity for the $F_{n, i}$, closely related to the definition equation of the $Y$ polynomials (Bell, loc. cit. p. 269), namely

Received by the editors February 15, 1946.

1 Numbers in brackets refer to the Bibliography at the end of the paper. 


$$
e^{-y} D_{x}^{n} e^{y}=Y_{n}\left(y_{1}, \cdots, y_{n}\right), \quad y_{s}=D_{x}^{*} y .
$$

Indeed

$$
Y_{n}\left(a g_{1}, \cdots, a g_{n}\right)=\sum_{i=1}^{n} F_{n, i}\left(g_{1}, \cdots, g_{i}\right) a^{i}
$$

and, by combination of this with (2),

$$
F_{n}=Y_{n}\left(a g_{1}, \cdots, a g_{n}\right), \quad\left(a^{i} \equiv f_{i}\right) .
$$

For concreteness, the first few $Y$ 's are listed as follows:

$$
\begin{gathered}
Y_{0}=1, \quad Y_{1}=y_{1}=a g_{1}, \quad Y_{2}=y_{2}+y_{1}^{2}=a g_{2}+a^{2} g_{1}^{2}, \\
Y_{3}=y_{3}+3 y_{2} y_{1}+y_{1}^{3}=a g_{3}+3 a^{2} g_{2} g_{1}+a^{3} g_{1}^{3} .
\end{gathered}
$$

Recurrence and other relations for the $F_{n}$-before the identification $a^{i}=f_{i}$-may then be taken from Bell with slight changes for the change in arguments. The more important ones are as follows:

$$
\begin{aligned}
F_{n+1} & =\left(a g_{1}+D_{x}\right) F_{n} \\
& =a g(F+g)^{n}=\sum_{i=0}^{n}\left(\begin{array}{c}
n \\
i
\end{array}\right) a g_{i+1} F_{n-i} \\
& =\left(a g_{1}+\sum_{i=1}^{n} g_{i+1} \frac{\partial}{\partial g_{i}}\right) F_{n}, \\
\exp t F & =\exp a\left[\exp t g-g_{0}\right],
\end{aligned}
$$

where the last is symbolic and equivalent to

$$
F_{0}+t F_{1}+t^{2} F_{2} / 2 !+\cdots=\exp a\left[t g_{1}+t^{2} g_{2} / 2 !+\cdots\right],
$$

and to diBruno's formula

$$
F_{n}=\sum \frac{n ! a^{\sigma}}{s_{1} ! \cdots s_{n} !}\left(\frac{g_{1}}{1 !}\right)^{s_{1}} \cdots\left(\frac{g_{n}}{n !}\right)^{s_{n}}
$$

with summation over all non-negative integral solutions of $n=\sum i s_{i}$ and $\sigma=\sum s_{i}$.

Instances of (7) with special choices of $g$ may be used for verification of the numerical coefficients of $F_{n}$. Thus, with $g_{i}=1$, all $i$,

$$
\exp t F=\exp a\left(e^{t}-1\right)
$$

and

$$
F_{n}=\sum S_{i, n} a^{i}, \quad g_{i}=1,
$$


with $S_{i, n}=\Delta^{i} 0^{n} / i$ ! the Stirling number of the second kind, showing that the sum of numerical coefficients of $F_{n, i}$ is $S_{i, n}$. Again with $g_{1}=0$, $g_{i}=1, i>1$,

and

$$
\exp t F=\exp a\left(e^{t}-1-t\right)
$$

$$
F_{n}=\sum C_{i, n} a^{i}, \quad g_{1}=0, g_{i}=1, i>1,
$$

with $C_{i, n}$ an associated Stirling number with recurrence

$$
C_{i, n+1}=i C_{i, n}+n C_{i-1, n-1} \text {. }
$$

These numbers have a combinatorial interpretation similar to that for $S_{i, n}$, namely, $C_{i, n}$ is the number of rhyme schemes of $n$ verses with $i$ rhymes, such that each rhyme appears at least twice. This kind of verification of course may be extended at will. These results are in agreement with results of Wall [8] and Opatowski [5].

3. Function of many functions. The essentials of the general case are in the two function case

$$
F(x)=f[g(x), h(x)]
$$

If we write

$$
f_{i j}=\left[\frac{\partial^{i}}{\partial u^{i}} \frac{\partial^{j}}{\partial v^{j}} f(u, v)\right]_{u=g(x), v=h(x)}
$$

the first two derivatives are

$$
\begin{aligned}
& F_{1}=f_{01} g_{1}+f_{10} h_{1}, \\
& F_{2}=f_{20} g_{1}^{2}+2 f_{11} g_{1} h_{1}+f_{02} h_{1}^{2}+f_{10} g_{2}+f_{01} h_{2},
\end{aligned}
$$

and in general

$$
F_{n}=\sum_{i} \sum_{j} f_{i j} F_{n, i j}\left(g_{1} \cdots g_{i} ; h_{1} \cdots h_{j}\right) .
$$

Then as before the generating function for coefficients $F_{n, i j}$ is $\exp (-a g+b h) D_{x^{n}} \exp (a g+b h)$ and

$$
\begin{array}{rlr}
F_{n} & =Y_{n}\left(a g_{1}+b h_{1}, \cdots, a g_{n}+b h_{n}\right), & a^{i} b^{j}=f_{i j}, \\
\exp t F & =\exp \left[a\left(\exp t g-g_{0}\right)+b\left(\exp t h-h_{0}\right)\right] . &
\end{array}
$$

The last shows that (cf. Bell [1, equations 4.9 and 7.5])

$$
F_{n}=(G+H)^{n}=\sum\left(\begin{array}{c}
n \\
i
\end{array}\right) G_{n-i} H_{i}
$$


with

$$
G_{n}=Y_{n}\left(a g_{1}, \cdots, a g_{n}\right), \quad H_{n}=Y_{n}\left(b h_{1}, \cdots, b h_{n}\right) .
$$

The numerical coefficients associated with $Y_{n}$ then reappear here and in the general case, as noted by Opatowski [5], who used the generalization of diBruno's formula given by Teixeira [7]; compare also Dederick [2].

The extension of (12) and (14) to the general case is purely a matter of notation, which should be sufficiently evident.

4. Function of a function of a function. For completeness, we note briefly another extension to a function of a function of a function, which contains the essence of a general extension. Take

$$
F=f[g(h(x))] \text {. }
$$

Then

$$
\begin{aligned}
F_{n} & =Y_{n}\left(f G_{1}, \cdots, f G_{n}\right), & f^{i} \equiv f_{i}, \\
& =Y_{n}\left(\Gamma h_{1}, \cdots, \Gamma h_{n}\right), & \Gamma^{i} \equiv \Gamma_{i},
\end{aligned}
$$

with

$$
\begin{array}{rlrl}
g_{n} & =Y_{n}\left(g h_{1}, \cdots, g h_{n}\right), & & g^{i} \equiv g_{i}, \\
\Gamma_{n}=Y_{n}\left(f g_{1}, \cdots, f g_{n}\right), & & f^{i} \equiv f_{i} .
\end{array}
$$

The last two expressions exemplify a kind of associative equality which permits writing the general result in a variety of ways.

\section{BIBLIOGRAPHY}

1. E. T. Bell, Exponential polynomials, Ann. of Math. vol. 35 (1934) pp. 258-277.

2. L. S. Dederick, Successive derivatives of a function of several functions, Ann. of Math. vol. 27 (1925-26) pp. 385-394.

3. A. Dresden, Derivatives of composite functions, Amer. Math. Monthly vol. 50 (1943) pp. 9-12.

4. K. Menger, Algebra of analysis, Notre Dame Mathematical Lectures, No. 3, Notre Dame, Indiana, 1944.

5. I. Opatowski, Combinatoric interpretation of a formula for the nth derivative of a function of a function, Bull. Amer. Math. Soc. vol. 45 (1939) p. 944.

6. O. Schlömilch, Compendium der höheren analysis, s. 4, vol. 2, Braunschweig, 1879.

7. F. G. Teixeira, Sur les dérivées d'ordre quelconque, Giornale di Matematica di Battaglini vol. 18 (1880) p. 306.

8. H. S. Wall, On the nth derivative of $f(x)$, Bull. Amer. Math. Soc. vol. 44 (1938) pp. $395-397$.

Bell Telephone Laboratories 